\title{
Testing for Photometric Variability at the L/T Boundary
}

\author{
Melissa L. Enoch \\ Caltech, MS 105-24, Pasadena, CA, 91125
}

Michael E. Brown

Caltech, MS 150-21, Pasadena, CA, 91125

Adam J. Burgasser

Hubble Fellow; UCLA, 8965 Math Sciences Bldg, Los Angeles, CA, 90095

\begin{abstract}
We construct $K_{s}$-band light curves for nine field $\mathrm{L}$ and $\mathrm{T}$ brown dwarfs using the Palomar 60 inch Telescope. Results of a robust statistical analysis indicate that about half the targets show significant evidence for variability. Two of these variable targets have marginally significant peaks in the Lomb-Scargle periodogram. The phased light curves show evidence for periodic behavior on timescales of about 1.5 and 3.0 hours.
\end{abstract}

\section{Introduction}

With the continual discovery of many new sub-stellar objects of $L$ and $T$ spectral types, there is growing evidence that the transition between these classes, where the $J-K$ colors change from red to relatively blue, occurs over a very small range in effective temperature (Kirkpatrick et al. 2000). It has been suggested that this transition may be due to a change in cloud structure, in which case objects near the transition would exhibit significant photometric and/or spectroscopic variability (Burgasser et al. 2002).

Previous work has found several M and L brown dwarfs to be photometrically variable in the optical (Tinney \& Tolley 1999; Bailer-Jones \& Mundt 2000; Martín, Zapatero Osorio, \& Lehto 2001), but no dwarfs later than L5 were included in these samples. In an effort to better understand variability around the $\mathrm{L} / \mathrm{T}$ boundary, we have undertaken a program to monitor nine late $\mathrm{L}$ and early $\mathrm{T}$ field dwarfs.

\section{Observations \& Results}

Observations were made in the $K_{s}$-band $(\lambda=2.17 \mu \mathrm{m})$ with the Infrared Camera at the Palomar 60 inch Telescope. Each target was observed 2-5 times per night on 2001 September 30 - October 6 and October $25-28$, with 18 images taken at each observation. A relative magnitude $\delta m$ is calculated between the target and 
a reference star for each image. We assume that no variation is taking place on 10 minute timescales, and construct the light curve using $\Delta m=\sum_{i=1}^{18} \delta m$. The error in $\Delta m$ is computed from the scatter in $\delta m$ and Poisson statistics.

Table 1. Results of the robust variability analysis

\begin{tabular}{lccccc}
\hline & \multicolumn{3}{c}{ TARGET } & \multicolumn{2}{c}{ REFERENCE } \\
Target name & type & $\widetilde{\eta}$ & conf & $\widetilde{\eta}$ & conf \\
\hline 2MASS 2208+2921 & L2p & 0.79 & $44 \%$ & 0.84 & $66 \%$ \\
2MASS 0103+1935 & L6 & $\mathbf{1 . 0 6}$ & $\mathbf{9 8 \%}$ & 0.84 & $67 \%$ \\
2MASS 0205-1159 & L7 & 0.80 & $48 \%$ & - & - \\
2MASS 0030-1450 & L7 & $\mathbf{1 . 0 0}$ & $\mathbf{9 5 \%}$ & 0.78 & $44 \%$ \\
2MASS 0328+2302 & L8 & $\mathbf{1 . 2 1}$ & $>\mathbf{9 9} \%$ & - & - \\
SDSS 0423-0414 & T0 & 1.01 & $96 \%$ & $\mathbf{1 . 1 8}$ & $>\mathbf{9 9} \%$ \\
SDSS 0151+1244 & T1 & $\mathbf{0 . 9 9}$ & $\mathbf{9 5 \%}$ & 0.77 & $37 \%$ \\
2MASS 2254+3123 & T5 & $\mathbf{0 . 9 9}$ & $\mathbf{9 8 \%}$ & 0.82 & $59 \%$ \\
2MASS 0559-1404 & T5 & 0.80 & $47 \%$ & 0.80 & $52 \%$ \\
\hline \hline
\end{tabular}

To determine if any of the targets show evidence for variation we examine the robust median statistic, $\eta=\sum_{i}\left|\frac{\Delta m_{i}-\text { median }(\Delta m)}{\sigma_{i}}\right|$. We choose a robust method as it is much less sensitive to non-Gaussian errors than the $\chi^{2}$ test. A value of $\tilde{\eta}=\eta / d \geq 1$, where $d$ is the number of degrees of freedom, indicates likely variability. Monte Carlo simulations are done to quantify this likelihood. We take a confidence above $95 \%$ to indicate substantial evidence for variability. Results of the robust analysis appear in Table 1. About half of the targets, and one pair of reference stars, show significant evidence for variability as indicated by boldfaced type. 2MASS $0328+2302$ has only one reference star in the field, so we cannot be sure that the variability is intrinsic to the brown dwarf.

We check for periodic variations by constructing the the Lomb-Scargle weighted periodogram for unevenly sampled data (Woodward 1992). The confidence of a given peak is determined by the percentage of times that the same data, randomly ordered so that no periodic behavior can be present, produce a periodogram peak higher than the real value. There are no detected periods with a confidence $>95 \%$ for any of the objects; only two of the targets, 2MASS $0030-1450$ and SDSS $0151+1244$, have periodogram peaks above the $68 \%(\approx 1 \sigma)$ level. The phased light curves of these two objects suggest that periodic behavior is present on timescales of 1.5 and 3.0 hours respectively.

\section{References}

Bailer-Jones, C. A. L. \& Mundt, R. 2001, A\&A, 367, 218

Burgasser, A. J., et al. 2002, ApJ, 571, L151

Kirkpatrick, J. D., et al. 2000, AJ, 120, 447

Martín, E. L., Zapatero Osorio, M. R., \& Lehto, H. J. 2001 ApJ, 557, 822

Tinney, C. G. \& Tolley, A. J. 1999, MNRAS, 304, 119

Woodward, R. C. 1992, Ph.D. dissertation, University of Wisconsin, Madison 